\title{
Abstracts of Note: The Bioethics Literature
}

\begin{abstract}
This section is meant to be a mutual effort. If you find an article you think should be abstracted in this section, do not be bashful-submit it for consideration to feature editor Kenneth V. Iserson care of $C Q$. If you do not like the editorial comments, this will give you an opportunity to respond in the letters section. Your input is desired and anticipated.
\end{abstract}

Potter BK, O'Reilly N, Etchegary H, Howley $\mathrm{H}$, Graham ID, Walker M, et al. Exploring informed choice in the context of prenatal testing: Findings from a qualitative study. Health Expectations 2008;11:355-65.

This qualitative study, guided by concepts from the multidimensional measure of informed choice (MMIC), measured whether women in Ottawa, Ontario, made informed choices about prenatal testing. The study examined how each participant considered the relevant technical information in conjunction with her personal values. Using structured interviews with participants, the authors found that most women made an informed choice about whether to obtain prenatal testing.

Many women who made informed choices often focused on their views about pregnancy termination. Women who felt abortion was unacceptable made informed decisions to forgo prenatal testing because the information was of little or no value to them. Women who made informed decisions also obtained their knowledge from previous pregnancies, personal research, discussions with family or friends, and nondirective conversations with their healthcare provider. They tended to reflect on the personal consequences of the testing decision. In contrast, women who made uninformed choices about prenatal testing either thought that prenatal testing was routine medical care or felt that they did not have a choice. In some of these situations, the decision whether to pursue prenatal testing seemed inconsistent with the woman's expressed values. Women who made uninformed choices also did not seem to think through the testing decision in a personalized or self-reflective manner.

Obstetricians and other healthcare providers should promote active decisionmaking about prenatal testing. Healthcare providers should make certain that women understand they have a choice about whether to engage in prenatal testing. Conversations about the technical aspects of prenatal testing should be complemented by a discussion about the personal implications of prenatal testing. The authors also recommend that healthcare providers use decision aids that have been developed for prenatal testing. These aids encourage women to consider their personal values when deciding whether to obtain a prenatal test.

Boissy AR, Ford PJ, Edgell RC, Furlan AJ. Ethics consultations in stroke and neurological disease: A 7-year retrospective review. Neurocritical Care 2008;9:394-9.

Neurologic-specific treatment units are a relatively recent advancement in hospital care. Neurologic-focused units may give rise to unique ethical considerations because neurological disease often impacts functioning, cognition, and quality of life. There is currently little data related to how ethics consultation services are used in such arenas and what impact they have on patient care. The study retrospectively reviewed all ethics consultations obtained at the Cleveland Clinic between 1998 and 2004 involving neurological and neurosurgical patients. Fortynine consultations were identified. Physicians initiated ethics consultations in $84 \%$ of the cases, with consultations being equally shared by neurosurgeons, neurologists, and neurointensivists. Stroke $(n=26)$ and brain tumor $(n=7)$ were the most frequent diagnoses of patients referred for ethics consultation. Withdrawal of life support/futility $(n=15)$, conflict $(n=8)$, and capacity evaluations $(n=7)$ were the most common reasons ethics consultations were requested. The primary reasons for consultation did not differ between stroke and nonstroke patients. However, a subgroup analysis of withdrawal of life support cases showed that death by neurological criteria was more frequently a concern in stroke patients, whereas requests for aggressive care despite 
poor prognosis more commonly prompted consultation in nonstroke patients. Methods of resolution included facilitation of communication $(n=17)$, validation of physician decisions $(n=12)$, giving advice $(n=7)$, providing policy information $(n=7)$, and recommending additional services $(n=3)$. One case was referred for full ethics committee review, and in three cases, the ethics consultant served no clear function. In 53\% of cases, the ethics consultant arranged and led family meetings. Primary attending staff participated in half of such meetings. The study suggests that withdrawal of life support, declarations of death by neurological criteria, opinions about prognosis and treatment goals, decisional capacity, and surrogate decisionmaking are potential sources of confusion and conflict in the care of neurological patients. Though ethics consultations are often requested in such instances, the exact responsibilities and objectives of the consultant remain unclear. In the study, facilitation of communication and validation of physician decisions were two primary tasks of the consultant. Further clarification of the ethics consultant's role in neurological populations may lead to improved care of patients and families dealing with neurological disease.

McDonald KE, Keys CB. How the powerful decide: Access to research participation by those at the margins. American Journal of Community Psychology 2008;42(1-2):79-93.

The focus of the authors' study was on how members of institutional review boards and researchers make decisions regarding proposed research involving intellectually disabled adults. People with intellectual disabilities have, in the past, been involved in abusive research projects, such as the hepatitis studies at Willowbrook. Ethical violations regarding research participants in the past few years have led to vigorous public and professional debate as well as some government regulation regarding research subjects. The decisionmakers may view intellectually disabled adults as largely incompetent and therefore significantly restrict their access to research. This restriction may protect these vulnerable adults from exploitation but limits their ability to make choices. People with intellectual disabilities have struggled for decades to be fully active and as autonomous as possible in whatever community they choose to live. To exclude adults with intellectual disabilities from being research participants limits these individuals' opportunity to participate in a process that may benefit them as well as others with similar problems. The subjects for this study included researchers who had recently published on topics in the social sciences with intellectually disabled adults and institutional review board (IRB) members at the same institutions where the above researchers were conducting their work. Study subjects included 116 IRB members, 114 researchers whose studies involved recruiting people with intellectual disabilities, and 30 individuals who were both. The study participants were sent vignettes of four fictitious research studies, two of which included adults with intellectual disabilities and two of which did not include adults with intellectual disabilities. Each vignette described proposed research with little potential harm or with more potential harm. The vignettes were based on recently published research, and decisionmakers were asked to review them, assuming they would subsequently be providing ethical guidance to a graduate student or a colleague. Study participants then answered questions about the abilities of individuals in the vignettes to provide competent consent, the degree of risk they were exposed to, and the level of necessary safeguards or protections they needed. The study revealed that intellectual disability researchers in comparison to IRB members perceived less ability to consent and a greater need for protection of intellectually disabled participants, especially when there was more potential for harm. The study showed that the study participants' attitudes about people with intellectual disabilities would likely compromise access to participation in research of adults with intellectual disabilities. The authors suggest the creation of guidelines for best practices for inclusion of people with intellectual disabilities in research studies that are sensitive to their vulnerabilities and their desire for control over their lives. Such guidelines and other interventions such as having people with intellectual disabilities participate in IRBs could lead to more positive attitudes toward adults with intellectual disabilities and promote appropriate and equitable access to research for people with intellectual disabilities.

Briggs CL, Mantini-Briggs C. Confronting health disparities: Latin American social medicine in Venezuela. American Journal of Public Health 2009;99(3):549-55. 
Providing healthcare to the poor of Latin America has been a challenge since the early 20th century. Rooted in 19th-century European social medicine, Latin American social medicine became established in Chile, Argentina, and Ecuador in the early 20th century and spread to other areas of Latin America by the 1960s and 1970s. Collective rather than individual approaches to healthcare are emphasized in this approach to healthcare delivery. Economic and social determinants of health and factors that produce health inequities are emphasized in this paradigm. Health gains that had been achieved in Venezuela from these early social medicine initiatives diminished in the late 20th century, as reflected in declining spending on healthcare and a decaying health infrastructure. Healthcare expenditures were primarily from the private sector, and poor Venezuelans faced large fees for healthcare and medications that they could not afford. By the end of the 20th century, a substantial proportion of the Venezuelan population effectively had no access to healthcare. The left-leaning government of Hugo Chávez Frias in 1999 attempted to address these health disparities by translating Latin American social medicine principles into policies and practices. Modeled on the needs of a poor community in Caracus, Mission Barrio Adentro was established. Local residents emphasized healthcare as their greatest concern and discussed the practical barriers they confronted in obtaining adequate medical treatment. Community leaders, residents, and government representatives participated in discussions on how to bring healthcare to residents living in poor neighborhoods. One of the conclusions of these discussions called for physicians to live and work in the poor neighborhoods. Few Venezuelan physicians wanted to live in the barrios, but a deal was struck to have Cuban doctors provide this type of care in exchange for oil for Cuba from Venezuela. The Cuban doctors were trained in a form of medicine emphasizing familial, community, and environmental factors. Community workers who were poor but had managed to attend college assisted residents in forming neighborhood health committees. The Cuban doctors lived in homes in the barrios among the poor. The clinics they worked in were often no more than someone's home that had been converted for use as a makeshift clinic. By 2006, Mission Barrio Adentro included 23,789 Cuban doctors, dental specialists, optometrists, nurses, and other personnel and more than 6,500 sites where patients were seen. In addition to these primary care clinics, diagnostic centers, high technology centers, and rehabilitation centers were developed, along with new public hospitals, to meet the healthcare needs of the poor that could not be addressed in a primary care clinic. The authors' study involved interviewing 221 residents, 41 health professionals, and 28 government employees and included systematic observations at neighborhood clinics. Researchers accompanied health professionals or members of the local health committees on household visits. The research also involved surveying 270 heads of households, sampling every 10th house in blocks selected at random. The results of this study demonstrate that addressing healthcare disparities requires combining research and theory with the underserved communities' active involvement in designing solutions. Top-down and bottom-up perspectives are inadequate, and a more horizontal approach appears to be more effective. Local health committees played a crucial role in shaping the Mission Barrio Adentro and allowed for creative, flexible problem-solving approaches. Many of the individuals who helped design the Mission Barrio Adentro came from underserved communities but had obtained university training and were able to negotiate between the poor people in the communities that needed access to healthcare and healthcare professionals. Egalitarian clinical interactions between the Cuban physicians and Venezuelan patients were important factors that led to positive local perceptions and a willingness of poor people to become patients of Mission Barrio Adentro neighborhood clinics.

These Abstracts of Note were compiled by

Barry Morenz, Aimee Kaempf, and Kristen Rabe Smolensky. 